\title{
Cross Layer Design for Cooperative Transmission in Wireless Sensor Networks
}

\author{
Kanojia Sindhuben Babulal, Rajiv Ranjan Tewari \\ Department of Electronics and Communication, J.K Institute of Applied Physics and Technology, \\ University of Allahabad, Allahabad, India \\ E-mail: \{sindhukanojia, tewari.rr\}@gmail.com \\ Received April 19, 2011; revised June 1, 2011; accepted June 9, 2011
}

\begin{abstract}
Several protocols and schemes have been proposed to reduce energy consumption in Wireless Sensor Networks (WSNs). In this paper we employ farcoopt, a cross layer design approach with the concept of cooperation among the nodes with best farthest neighbor scheme to increase the Quality of Service (QoS), reduce energy consumption, increases performance and end-to-end throughput. We present cooperative transmission to connect previously disconnect parts of a network thus overcoming the separation problem of multi-hop network. We show that this approach improves connectivity over 50\% compared to multi-hop approaches and reduces the number of nodes necessary to provide full coverage of an area up to $35 \%$. Simulation results show that on increase of data rates i.e. packet the network life time increases in farcoopt as compared to traditional multi hop approach. The result of this analysis is presented in this work.
\end{abstract}

Keywords: Cooperative Network, Cross Layer Design, Wireless Sensor Networks, Energy Saving, Communication Protocol, Routing

\section{Introduction}

During last few years processor power consumption has increased by over $200 \%$ every four years, while battery energy density has increased by modest 25\% [1]. In Wireless Sensor Network (WSNs), sensor nodes are deployed in a hostile environment which comes up with the problem charging the battery supported equipments. In fact it has been observed that power is and will always remain a problem to be solved. To deal with this problem, there have been increasing interests in design for wireless networks that rely on interactions between various layers of the protocol stack. This approach called cross layer design has been widely recognized as a promising solution for various problems in wireless networks. However, such a design principle across different layers usually involves high complexity [2]. Cross layer design is currently one of the most active research areas in computer networks. Cross layering means allowing communication of layers with any other layer [3]. Cross Layering was thought of to address QoS (Quality of Service), Poor performance, wireless links, mobility, packet loss, delay problems observed in wireless networks. The traditional method to forward data in sensor network is multi hop routing. Many MAC protocols and routing algorithm for WSN are developed like PAMAS [4], SMAC [5]. Many network layer algorithms such as MTE [6], DSR [7], and AODV [8] try to decrease the total energy consumption by multi hop routing. Nodes try to find a route between source and a destination to forward the information. For communication at least one route must exist. If two nodes are not able to communicate to each other because they are too far away to send/receive RF signals the network is segmented [9].

\section{Related Works and Background}

In research paper [10] an energy efficient routing algorithm is proposed which is based on the concept of switching transmission power level based on the volume of data. It proposes a back off mechanism by switching OFF unintended receivers based on the power of the received radio signal. In [11], the cross layer interaction is shown with MAC and the routing layer. The cross layer design is implemented in this paper by showing the interaction of 802.11 MAC protocol and Dynamic Source Routing (DSR) protocol. This design was able to reduce the routing overheads by decrementing the route man- 
agement process performed by the DSR protocol. In paper [12], an energy optimization protocol based on cross layer wireless sensor networks named as EOA was proposed. For this physical, MAC and the routing layer were considered. A feedback algorithm is proposed which computes proper transmission power level between nodes was computed. Then the EOA routing protocol can make use of the transmission power as a metric by choosing route with optimal power consumption to forward packets. In research paper [13] an efficient flooding scheme which reduce the re-transmission redundancy are introduced. In this node can decide if it should retransmit the broadcast packet or not. In probabilistic flooding, node re transmits the broadcast packet according to probability. In [14] energy efficient cross layer routing is obtained by combining the MAC functionality with the routing decision at the network layer. By this the overhead is reduced to some level. The protocol used is based on geographic routing and the end-to-end decision is made by the source node and the destination node.

In [15] a cross layer design frame work called CoopGeo, which performs the greedy forwarding nodes that wins the contention to forward the message, cooperative relaying scheme with single relay selection mechanism where the source node and relay node jointly transmit data through wireless channel is used. CoopGeo gains physical layer performance in terms of reliability. In [16, 17] authors understand cooperative transmission in the sense that several sensor nodes transmit symbols simultaneously to achieve the power gain. In [17] the broadcast coverage of a system using cooperative transmission is analyzed. It is based on a continuum approach modeling the nodes as a homogenous density of possible transmits power. This simplifies the modeling and leads to closed-form solution and formulations. In [18] there is also a small section on the coverage achievable with cooperative transmission.

Our work is based on the maximum intermediate node concept [18-21]. In these researchers, the authors' proposed novel concept for routing i.e. instead of using the traditional method for routing they introduced a new concept of sending the data to the maximum distance intermediate node. In [19] they have also applied a dynamic re-transmission scheme also. And an important thing to note is that in those they have considered the networks that are static or less mobile. In our paper we have talked about the network that is mobile in nature, and applied cooperative transmission scheme which helps to increase the QoS and reliability of the system.

The rest of the paper is organized as below: Section 3 discusses briefly about the cooperative transmission scheme. Section 4 presents the detail of the network model that is used in WSN and the detail description of FARCOOPT scheme. Section 5 shows the simulation of the scheme. Section 6 discusses the result of the simulations.

\section{Network Model \& Cooperative Transmission Theme}

Cooperative transmission theme is applied to the networks that are not able to communicate with each other. It is in ideal means applied to tackle the bad connectivity. Connection break can be from any of the reasons like random installation process, changes in the environment, wear-out or due to the mobility of the networks. With cooperative transmission, a group of nodes can combine its emission power and achieve a higher emission power as a whole. To do so, cooperatively transmitting nodes emit identical symbols synchronously to superimpose the emitted waves on the physical medium. The destination receives the sum of waves and thus a higher total power. The more nodes cooperatively transmit the higher will the power on the physical medium be. With the higher power, the nodes can reach destinations that are very far away.

In this paper, we address the concern of cross layer design with wireless medium of physical layer and MAC sub layer being passed to the network layer and the information of network being transmitted to lower layers. We aim to improve interactions of physical, MAC, and network layers. Information about the physical channel condition is transmitted from physical layer to network layer. Data rate and power information are transmitted from network layer down to the physical interface.

We model the dynamic wireless model as a set of $N$ nodes distributed into two dimensional planes. Wireless network is represented as a graph $G(V, E)$, where $V=$ $\{v 1, v 2, v 3, \cdots, v n\}$ is a finite set of nodes and $E=\{e 1$, $e 2, e 3, \cdots, e n\}$ a finite set of links, the sink and nodes are randomly deployed in the areas. As wireless networks do not have fixed infrastructure, sensor nodes col-

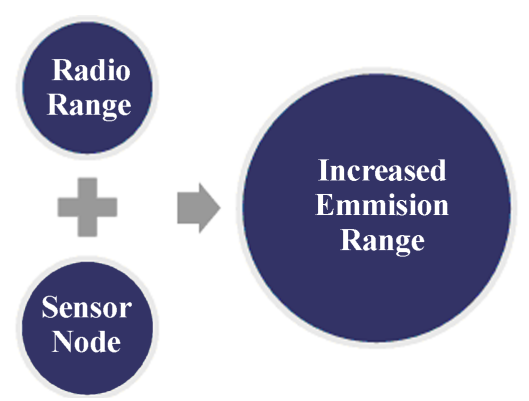

Figure 1. Increased emission range by summation of radio range. 
laborate to work together by wireless channel. Every node is aware of its location and also the location of the neighbor. The network is also mobile in nature.

The set of nodes source $V_{\text {source }}=\left\{V_{S 1}, V_{S 2}, \cdots, V_{S n}\right\}$ knows the destination location. In this network $V_{\text {source }}$ is sending a set of packets to the destinations $d(i)$ nodes. Each source node $i$ transmit to its destination node $d(i)$ which may or may or not belong to S. Let hij denote the channel gain power between node $i$ and $j$. all nodes are assumed to be energy constrained and the total energy of each node is denoted by $E^{\text {total }}$. Note that the energy allocated to a node is the total energy utilized in transmitting and relaying this node information. All nodes are fully charged initially and they have same energy. Since for a particular source destination (s-d) pair, some nodes may be far away from both source and destination only neighbor are selected in order to increase power efficiency and avoid error propagation.

We assume $A_{t x}$ as the nominal transmit power of a node. We assume that the transmit power is same for all nodes.

$A_{r x, j} \leftarrow i$, is the received power of a signal propagated from node i to node j. a receive power $P_{r x, j} \leftarrow i$, above a given threshold $P_{\text {th }}$ will provide sufficient SNR in receiver to decode the transmission. For cooperating transmission scheme power gain can be completely exploited. A group $\mathrm{G}$ of nodes all connected to each other can combine their power and transmits toward a node $j$ :

$$
P_{r x, j} \leftarrow G=\sum_{i \in G} P_{r x, j} \leftarrow i
$$

In our wireless communication system at physical layer we consider path loss model. Path loss model is the difference between the transmitted power and the received power. It represents signal l level attenuation caused by free space propagation, reflection, diffraction and scattering. Path loss in its simplest form can be described as below

$$
L=10 n \log _{10}(d)+C
$$

where $L$ is the path loss in decibels, $n$ is the path loss exponent, $d$ is the distance between the transmitter and the receiver, usually measured in meters, and $C$ is a constant which accounts for system losses

For radio and antenna consideration path loss can be calculated as below

$$
L=20 \log _{10(4 \pi d \mid \lambda)}
$$

where $L$ is the path loss in decibels, $\lambda$ is the wavelength and $d$ is the transmitter-receiver distance in the same units as the wavelength. For path loss which we model as a radial fading $\square 1 / \gamma_{i, j}^{\beta}$. With this the maximum distance for successful communication is $\gamma_{t h}^{\beta}=\frac{P_{t x}}{P_{t h}}$. For channel we assume Rayleigh fading. It models the effect of a propagation environment on a radio signal and assumes the magnitude of a signal that has passed through communication channel will randomly fade according to the Rayleigh distribution. Most mobile communication occurs when there is no direct path between the base station antenna and the mobile user. The signal reflects off many objects along the path between the two. This propagation follows a Rayleigh probability distribution about the mean signal level:

$$
\begin{aligned}
p_{r}(r) & =\frac{r}{\alpha^{2}} \exp \left(\frac{r^{2}}{2 \alpha^{2}}\right) \operatorname{prob}[r<R] \\
& =P_{R}(R)=1-\exp \left(-\frac{R^{2}}{2 \alpha^{2}}\right)
\end{aligned}
$$

$R$ is the signal level, $\alpha$ the value of the peak in the distribution, with mean $\alpha \sqrt{\pi / 2}=$ and median $R_{M}=$ $\alpha \sqrt{2 \ln (2)}=1.774 \alpha$. The antennas of wireless sensor nodes are trans-receiver, Omni directional behavior. We consider that transmission of a node takes a certain amount of energy $E_{t x}$. Hybrid cooperative communications consist of three parts: a single source $S$, a single destination $D$, and set of potential relay nodes $B$. A packet fails only when there is interference on intended receiver. Even if the packets collide partially they are considered to be collided. We assume that throughout the process there is some mechanism that notifies the sender of the success and failure of its transmission. We have assumed initially that the network is mobile in nature and no fixed infrastructure exit.

\section{Farcoopt Scheme}

Farcoopt is divided in two major subsections

(4.1) Sending the data

(4.2) Cooperative Transmission applied in case

(4.2.1) when retransmission occurs

(4.2.2) when link failure occurs

Whole process is explained as below. As we have assumed earlier that our network is mobile so the chances of link breakages also increases. Let us assume Figure 2. Case 4.1: want to send the data from source $S(1)$ to destination $D(10)$. First the shortest path from $S$ to $D$ is obtained by using algorithm all pair shortest path. Which is in this example set of nodes $\{1,2,3,6,7,9,10\}$. But

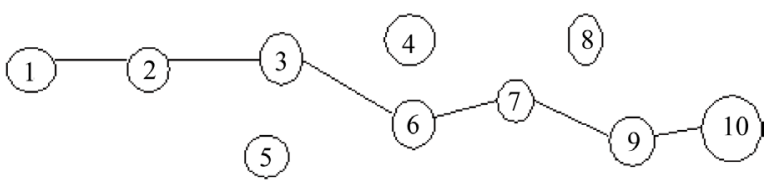

Figure 2. Network consists of 10 nodes where source (1) and destination (10). 
instead of using the simple multi hop (node by node) routing we use the best farthest neighbor concept. Best farthest neighbor can be assumed as that neighbor who is active (i.e. free to receive the data), charged, it is in transmission or radio range. The benefit of best farthest neighbor is that it reduces the overhead and instead of sending data to the node next with same energy why not to send to that node which is far not successfully receive the data, and to some extend it also help to save energy as while the nodes are communicating other nodes can sleep. This decreases the overhead. When any communication is going on between two nodes other neighbor nodes do not do any transaction i.e. Case $\mathbf{4 . 2}$ when cooperative transmission takes places they (best farthest neighbor) need to be active. As it is assumed that transmission of data is with same energy no matter what data has to be send. Before the sending of the data occurs it is assumed that procedure of sending the request to check the status of the best farthest neighbor, and on the receipt of the acknowledgement that it is free and would receive, then only data is send. It means that after the confirmation that node is active and free to receive; data is transmitted to that node.

When once the route is discovered $S(1)$ sends data to node 3 which is $S(1)$ best farthest neighbor. On successful delivery of data node 3 has to send back acknowledgement notifying that it has successfully receive the data. Case 4.2.1 If the acknowledgement is not received within fixed time $T f$, it is assumed that packet failure has occurred. Now we need to retransmit the data, so here we use the cooperative retransmission scheme and send the data to best farthest neighbor. As we are sending to the best farthest neighbor the number of relay nodes would be less which would help in cooperative transmission. To do so, cooperatively transmitting nodes emit identical symbol synchronously to superimpose the emitted waves on the physical medium. And then node 3 notifies with acknowledgement for successful delivery of data. These acknowledgement bits are small in size.

Case 4.2.2 Now node 3 s best farthest neighbor is node 7 , but during communication due to some reason (may be due to mobility, environment changes etc.) that link fails and network is cluster they are not able to communicate with each other, then also the cooperation strategy is applied to find the nearest link, let us suppose in this example it is node 8 which would help to join the two clustered network. The data that is still lying on node 3 has to be forward to node 8 . Node 8 also has to notify for successful receiving of the data. At this stage it should be made clear that node 8 would not be able to send back the notification for successful data delivery with the help of the cooperation means. This step would be costly in terms of time and energy because we are only sending the acknowledgement and not the data. But still it would have to be done. This would increase the (Quality of Service) QoS and also the reliability of the network. Important issue is that we are not proceeding without the guarantee that the data at that step was successfully received. But It can be noted that node 8 was not in the shortest path which we calculated before the starting of the process. Again the shortest path would be calculated and the data would be forwarded as discussed above. Benefits of best farthest neighbor is that, it reduces the overhead of control signal, and when same energy we can transmit at bode situated at farther node, then why to send it to just the neighbor.

\section{Simulations \& Results}

The set up consist of 250 sensor nodes which are randomly deployed in area $1000 \times 1000 \mathrm{~m}^{2}$. We summarize the configuration setting used as input in our simulator. Packets are generated by Poisson process. The mobile rate of nodes is $v$, we consider that maximum speed of 10 meter per seconds. We assume $\lambda_{e}$ as the average number of UDP packets generated per second for any source nodes and packets size of 1024 bytes. We change the value of $\lambda_{e}$ to show the performance of FARCOOPT. Network Lifetime is used as metric to evaluate the performance of cross layer design. We define network lifetime as the time taken for $50 \%$ of the sensor nodes in a network to drain up their power. Table 1 gives the details of the simulation parameters.

Figures 3-5 shows the comparison of 3 different schemes DSR, E2XLRADR, and FARCOOPT method. All the three schemes are build on different strategy.DSR uses traditional multi hop routing; E2XLRADR uses the concept of maximum intermediate node. And FARCOOPT used the concept of best farthest neighbor with cooperative transmission. We can observe that with the increase of the node number the lifetime of FARCOOPT increase in comparison to the other schemes. As the rate increases, the lifetime of DSR changes very rapidly because of interference, but our FARCOOPT approach can handle

Table 1. Simulation parameters.

\begin{tabular}{ll}
\hline Parameters & Value \\
\hline Simulation area & $1000 \times 1000 \mathrm{~m}^{2}$ \\
Number of nodes & $10, \cdots, 250$ \\
Transmission range (M) & 250 \\
Bandwidth (Mbps) & 2 \\
Max node speed (MIS) & 10 \\
$\mathrm{~T}_{\mathrm{f}}$ (Time fixed) & 100 \\
Data item size (KB) & $(1-10)$ \\
Number of data item & 1000 \\
Request interval (S) & 10 \\
Simulation time (S) & 2000 \\
$\lambda_{e 1}$ & 3packets/sec \\
$\lambda_{e 2}$ & 6packets/sec \\
$\lambda_{e 3}$ & 9packets/sec \\
\hline
\end{tabular}




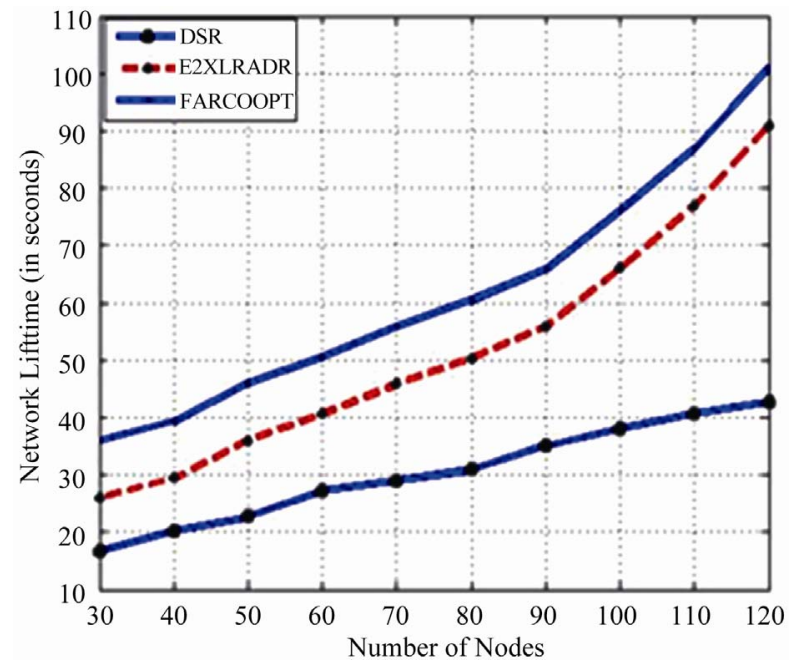

Figure 3. Network lifetime of 3 scheme at which node mobile rate is 3 packet/sec.

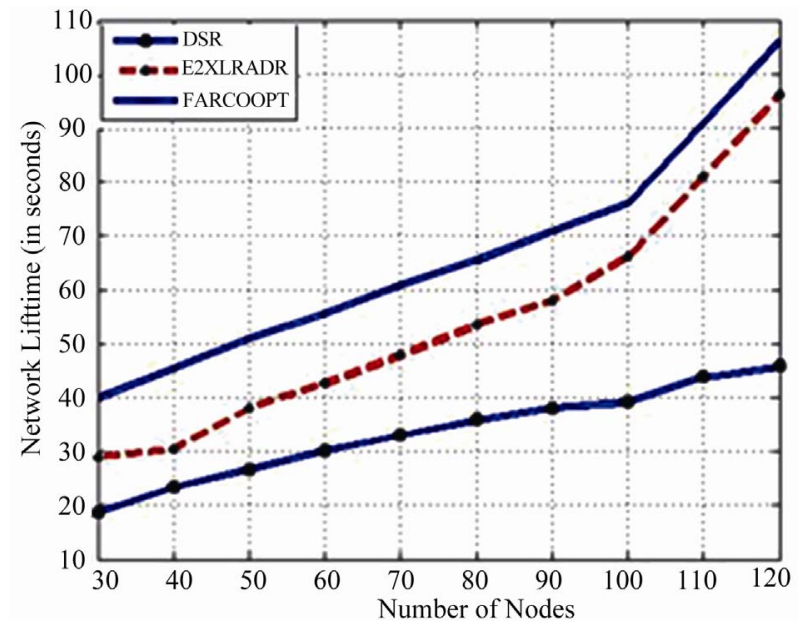

Figure 4. Network lifetime of 3 scheme at which node mobile rate is 6 packet/sec.

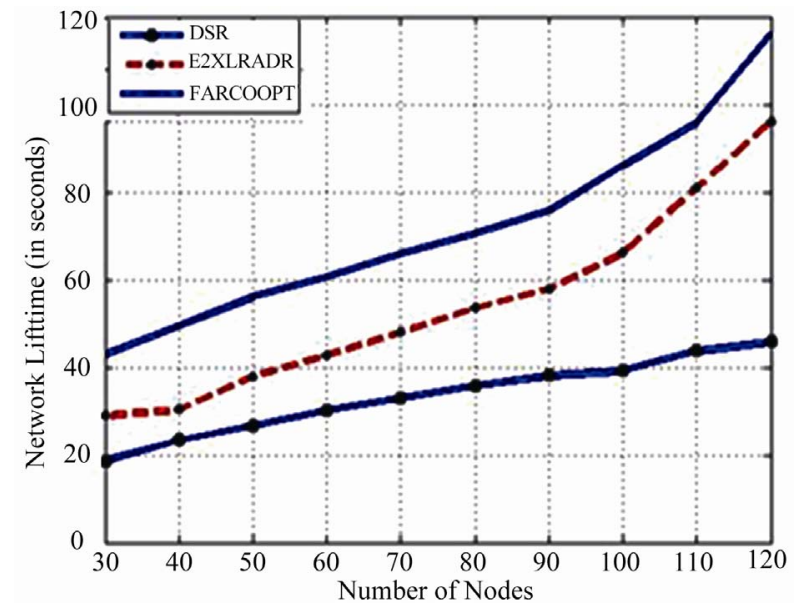

Figure 5. Network lifetime of 3 scheme at which node mobile rate is 9 packet/sec.

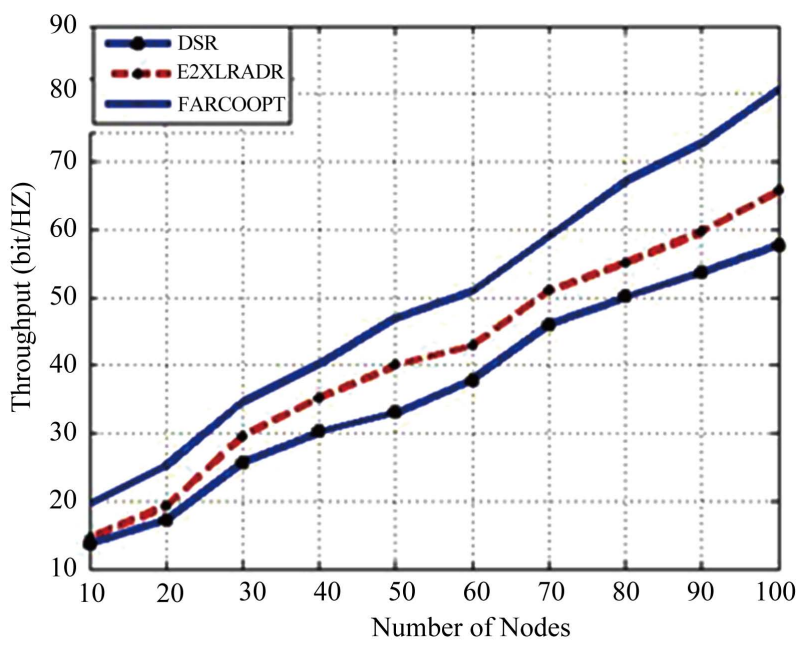

Figure 6. Increase in the throughput in FARCOOPT.

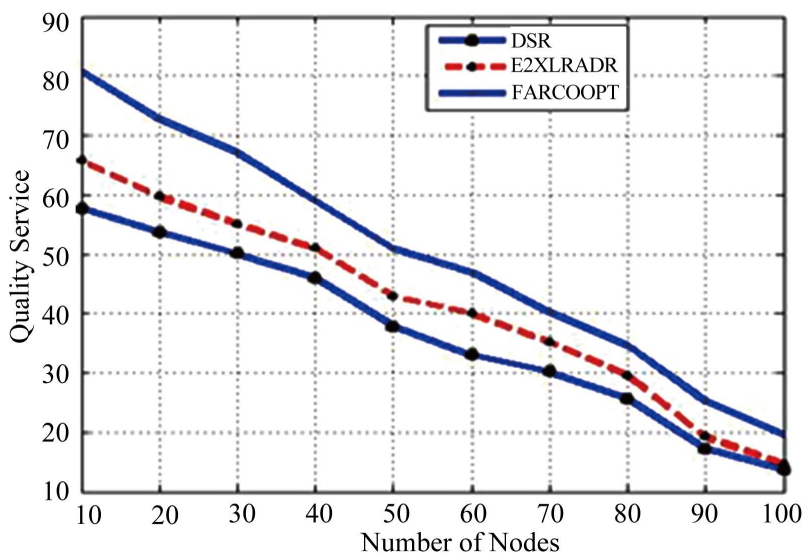

Figure 7. On increase of number of packet the quality of service increases.

with this situation. Figure 6 talks about the throughput in terms of time. Throughput is not degraded with increase in time of the process. Throughput is measured in bit/Hz which is the unit of frequency efficiency. Figure 7 investigates about the increase in Quality of service even if the number of packets increases.

\section{Conclusions}

Cross layering is the best approach for wireless sensor networks. In traditional layering network various protocol layers can only strictly communicate in "layer by layer” approach. In such conditions, layers are not designed to adapt to the changing environment. This leads to inefficient use of energy. We believe that cross layer design should alleviate this problem. We have used the concept of sending the data to the best farthest neighbor with the cooperative transmission theme. Cooperative retransmission has direct impact on the end-to-end delay 
throughput of WSN. We have used the cooperative retransmission when we have to retransmit the data and when during communication link failure occurs. We compared our scheme with traditional approaches like the DSR protocol. By this approach we achieve end to end packet delivery, less loss of data, reliability of network, QOS, fairness in service, and energy is also saved to some extent. Several power conservation schemes have been proposed in the literature for prolonging the lifetime of the sensor network, either by trying to reduce the number of retransmission through efficient routing or by taking the advantages of the sleep mode capabilities of the sensor node. Network Performance is also increased by FARCOOPT approach.

\section{References}

[1] K. Lahiri, A. Ragunathan, S. Dey and D. Panigrahi, "Battery-Driven System Design: A New Frontier in Low Power Design," Proceedings of 7th Asia and South Pacific and the 15th International Conference on VLSI Design, Bangalore, 7-11 January 2002, pp. 261-267.

[2] V. Kawadia and P. R. Kumar, "A Cautionary Perspective on Cross Layer Design,” IEEE Wireless Communications, Vol. 12, No. 1, 2005, pp. 3-11. doi:10.1109/MWC.2005.1404568

[3] V. Srivastava and M. Motawani, "Cross Layer Design: A Survey and the Road Ahead," IEEE Communications Magazine, Vol. 43, No. 12, 2006, pp. 112-119. doi:10.1109/MCOM.2005.1561928

[4] S. Singh and C. S. Raghavendra, "PAMAS: Power Aware Multi-Access Protocol with Signaling for Ad Hoc Networks," ACM Computer Communication Review, Vol. 28, No. 3, 1998, pp.5-26. doi:10.1145/293927.293928

[5] W. Ye, J. Heidermann and D. Estrin, "An energy Efficient MAC Protocol for Wireless Sensor Networks," Proceedings of IEEE Infocom, USC/Information Sciences Institute, New York, June 2002, pp. 1567-1576. http://www.isi.edu/johnh/PAPERS/Ye02a.html

[6] W. Heinzelman, A. Chandrakasan and H. Balakrishnan, "Energy Efficient Communication Protocol for Wireless Microsensor Networks," Proceedings of the Hawaii International Conference on System Sciences, Washington DC, January 2000, pp. 4-7. doi:10.1109/HICSS.2000.926982

[7] D. B. Johnson, D. A. Maltz, Y.-C. Hu and J. G. Jetchev, "The Synamic Source for Mobile Ad Hoc Wireless Networks,” Internet Engineering Task Force Internet Draft, 2000.

http://www.ietf.org/internetdrifts/draft-ietd-manet-adsr-0 $\underline{6 . t x t}$

[8] C. E. Perkins and E. M. Royer, "Ad Hoc on Demand Distance Vector Routing,” Internet Engineering Task Force Internet Draft, 2003. http://www.ietf.org/internetdrifts/draft-ietf-manet-aodv-1 $\underline{3 . t x t}$
[9] R. Gunesekaran and H. Qi, “XLRP: Cross Layer Routing Protocol for Wireless Sensor Network,” IEEE Communication Society, Las Vegas, 31 March-3 April 2008, pp. 2135-2140.

[10] N. Chilamkurti, S. Zeadally, A. Vasilakos, et al., "Cross Layer Support for Energy Efficient Routing in Wireless Sensor Networks,” Journal of Sensors, Vol. 2009, 2009, pp. 1-9. doi:10.1155/2009/134165

[11] Y. B. Bai, S. J. Liu, et al., "An Energy Optimization Protocol Based on Cross Layer for Wireless Sensor Networks," Journal of Communication, Vol. 3, No. 6, 2008, pp. 27-34.

[12] S. Ni, Y. Tseng, Y. Chen and J. Sheu, "The Broadcast Storm Problem in Mobile Ad Hoc Network," Wireless Network, Vol. 8, No. 2-3, 2002, pp. 153-167.

[13] P. Skraba, H. K. Aghajan and A. Bahai, "Cross Layer Optimization for High Density Sensor Network: Distributed Passive Routing Decisions, Ad Hoc, Mobile, and Wireless Networks," Proceedings of the Third International Conference, ADHOC-NOW, Vancouver, July 2004, pp. 266-279.

[14] T. Aguilar, et al., "A Cross Layer Design Based on Geographic Information for Cooperative Wireless Networks," Proceedings of the IEEE 71st Vehicular Technology Conference, Taiwan, 16-19 May 2010, pp. 1-5.

[15] A. Scaglione and Y. W. Hong, "Opportunistic Large Arrays: Cooperative Transmission in Wireless Multi Hop Ad Hoc to Reach Far Distances," IEEE Transaction on Signal Processing, Vol. 51, No. 8, 2003, pp.2082-2092. doi:10.1109/TSP.2003.814519

[16] A. Scaglione and Y. W. Hong, "Opportunistic Large Arrays: Cooperative Transmission in Wireless Multi Hop Ad Hoc to Reach Far Distances," IEEE Transaction on Signal Processing, Vol. 51, No. 8, 2003, pp.16-20. doi:10.1109/TSP.2003.814519

[17] K. S. Babulal and R. R. Tewari, "E2XLRADR (Energy Efficient Cross Layer Routing Algorithm with Dynamic Retransmission for Wireless Sensor Network”, International Journal of Wireless \& Mobile Network, Vol 2, No. 3, August 2010, pp.166-177. http://www.airccse.org

[18] K. S. Babulal and R. R. Tewari, "Cross Layer Energy Efficient Routing (XLE2R) for Prolonging Lifetime of Wireless Sensor Networks," Proceedings of IEEE International Conference on Computer and Communication Technology, Allahabad, 17-19 September, 2010, pp. 167-177.

[19] K. S. Babulal and R. R. Tewari, "Routing Algorithm: An Energy Efficient Sleep/Awake Cross Layer for Wireless Sensor Networks”, Advances in Wireless and Mobile Communications, Vol. 3, No. 2, 2010, pp. 139-146. http://www.ripublication.com/awmcv3n2.htm

[20] K. S. Babulal, T. R. Ranjan, “Cross Layer RouteDiscovery Using Farthest Intermediate Link Distance Reliability Cost Based for MANETS," National Academy of Letters, Vol. 34, No. 5-6, 2011.

[21] K. Albert, B. Michael, D. Christian and R. Till, "Increasing Connectivity in Wireless Sensor Network Using Cooperative Transmission," The 3rd International Conference on Networked Sensing Systems (INSS), Chicago, 31 May-2 June 2006. 